\title{
Melhorias de Gestão de Cursos a Distância Através da Análise de Acessos ao AVA
}

\author{
José Jorge L. Dias Jr. ${ }^{1}$, Ricardo Serrano² ${ }^{2}$ Eudisley dos Anjos ${ }^{3}$, Lucídio Cabral ${ }^{4}$, \\ Jan Edson Rodrigues Leite ${ }^{5}$, Estêvão Domingos Soares de Oliveira ${ }^{6}$, Hercilio de \\ Medeiros Sousa ${ }^{7}$. \\ ${ }^{1}$ Universidade Federal da Paraíba/Centro de Ciências Aplicadas e Educação \\ ${ }^{2}$ Universidade Federal da Paraíba/UFPB Virtual \\ ${ }^{3,4}$ Universidade Federal da Paraíba/Centro de Informática \\ ${ }^{5}$ Universidade Federal da Paraíba/Centro de Ciências Humanas, Letras e Artes \\ ${ }^{5,6}$ Universidade Federal da Paraíba/PPGI/UFPB Virtual \\ jorge@dce.ufpb.br, \{hercilio,jan.edson\} @virtual.ufpb.br, \\ ricardo@virtual.ufpb.br, eudisley@ci.ufpb.br, estevaodso@gmail.com
}

\begin{abstract}
The quality of distance education courses have had significantly increaseament in recent years. New technologies have allowed better use and management of Virtual Learning Environments (VLE). However, this improvement goes beyond technology and several challenges must still be overcome. This work presents an analysis of access to the VLE as a way of planning actions and strategies for the reduction of existing barriers in the management of distance education courses. Thus, this work uses a Business Intelligence (BI) tool for collecting and analyzing data to allowing to make better decisions in relation to infrastructure, resources and teaching strategies.
\end{abstract}

Resumo. A qualidade dos cursos de Educação A Distância (EAD) têm aumentado muito nos últimos anos. As novas tecnologias têm proporcionado um melhor uso e gestão dos Ambientes Virtuais de Aprendizagem (AVA). Entretanto, essa melhoria vai além da tecnologia e diversos desafios ainda devem ser superados. Este trabalho apresenta o uso da análise de acesso ao AVA como forma de planejamento de ações e estratégias para redução dos entraves existente na gestão dos cursos EAD. Para isso, foi utilizada uma ferramenta de Business Intelligence (BI) para coleta e análise de dados que permitam uma melhor tomada de decisões em relação a infraestrutura, recursos e estratégias de ensino.

\section{Introdução}

A Educação a Distância (EaD) surgiu desde 1728 com o professor Caleb Phillips e até hoje continua a crescer (Litto et al., 2009). No Brasil o crescimento mais significativo deu-se nos últimos 40 anos, quando a iniciativa privada passou a considerar o ensino superior como um interessante nicho de mercado, aumentando o número de vagas oferecidas nos cursos EaD. (Chamlian, 2003). Hoje, esta modalidade tornou-se um dos tópicos mais abordados em propostas e análises educacionais. O escopo tem abrangido desde sistemas de ensino, áreas de formação, treinamentos profissionais, acessibilidade, e muitas outras ações que multiplicam-se constantemente. 
De acordo com o último senso da ABED (Associação Brasileira de Educação a Distância) em 2012, o número de alunos brasileiros nos cursos EaD era de 5.436.243 (Litto et al., 2009). O perfil dos estudantes tem variado bastante e suas necessidades têm sido cada vez mais específicas. Isso deve-se principalmente a facilidade de acesso a internet e a grande gama de cursos e tecnologias oferecidas.

Uma das principais tecnologias que tem se tornado essencial na EAD são os Ambientes Virtuais de Aprendizagem (AVA) (Santos, 1999; Dillenbourg, et al., 2002). Esses ambientes são utilizados como principais plataformas de ensino a distância e possuem diversos atributos que elevam sua qualidade, tais como: segurança, usabilidade, performance, acessibilidade, flexibilidade, portabilidade, mobilidade, modularidade, manutenibilidade, entre muitos outros (Wickersham at al., 2006, Barbera, 2004). Portanto, a escolha do ambiente que melhor balanceia tais atributos é essencial para atender os requisitos do curso.

Além da escolha do AVA, é importante também que o gerenciamento dos recursos e funcionalidades seja realizado da melhor forma possível. Essas medidas ajudam a garantir um maior rendimento na execução das atividades oferecidas pelo curso e também, compreender melhor as necessidades dos usuários. (Beetham et al., 2007) Por isso, entender o comportamento dos usuários do AVA é uma importante estratégia visando a melhoria da gestão dos cursos a distância (Ike-Elechi et al., 2012). Entretanto, ainda há uma carência de ferramentas e técnicas que ajudem a gerenciar tais ambientes de forma satisfatória.

A UFPB Virtual é a unidade de educação a distância da UFPB (Universidade Federal da Paraíba) para dar suporte a 9 cursos de graduação e 2 cursos de pós graduação. São mais de $7 \mathrm{mil}$ alunos utilizando o AVA. Portanto, este trabalho apresenta um relato de experiência que buscar mostrar a importância de analisar os acessos ao AVA como uma forma de melhorar a gestão dos cursos a distância. Esta análise permite criar ações estratégicas em relação ao AVA, permitindo uma melhor alocação dos recursos, por exemplo.

A estrutura do trabalho está dividida da seguinte forma. Na Seção 2 detalhamos a metodologia utilizada para desenvolvimento deste projeto. A Seção 3 contém os dados obtidos e a análise que foi realizada sobre os dados, mostrando os principais resultados obtidos. Os trabalhos relacionados estão descritos na seção 4 e, finalmente, a Seção 5 apresenta algumas considerações finais e trabalhos futuros.

\section{Metodologia}

Para desenvolvimento deste trabalho, dividimos as atividades em três etapas principais: levantamento das informações para análise de acessos e definição de hipóteses, classificação dos dados e análise dos dados de acesso. Cada etapa será melhor descrita a seguir.

\subsection{Contexto da UFPB Virtual}

A UFPB Virtual está vinculada à Universidade Federal da Paraíba, e seu corpo discente é composto de aproximadamente 7000 alunos ativos. Sua atuação alcança 28 cidades de cinco estados da região nordeste. Atualmente, a instituição oferece vagas nos cursos de licenciatura a distância em Letras, Matemática, Pedagogia, Ciências Agrárias, Ciências Biológicas, Ciências Naturais e Letras/Libras, além de organizar cursos de capacitação e 
aperfeiçoamento, dentre outras atividades acadêmicas. No início de 2013, os novos cursos a distância de licenciatura em Computação e de bacharelado em Administração Pública foram iniciados.

A UFPB Virtual adotou o ambiente virtual de aprendizagem Moodle, uma plataforma de software livre de apoio ao ensino e aprendizagem com mais de 60 milhões de usuários, para viabilizar a maior parte das atividades acadêmicas exigidas pelos cursos a distância. Tais atividades são postadas e avaliadas pelos professores e tutores, e realizadas pelos alunos, de forma online através do Moodle. A interação entre os estudantes e mediadores de ensino também ocorre, na maioria das vezes, por meio de diversas ferramentas disponibilizadas pelo Moodle. Caso o aluno deseje auxílio presencial, há tutores presenciais que atuam nos polos de apoio dos municípios alcançados pela UFPB Virtual.

\subsection{Coleta de dados}

Para levantamento dos dados necessários para este projeto, utilizou-se Google Analytics. Esse é um serviço oferecido pelo Google que armazena o tráfego de um website e gera estatísticas detalhadas sobre esse. Através deste serviço é possível fazer rastreamento de usuários, marketing, visualização de diferentes tipos de relatórios, etc, funcionando como uma ferramenta de Business Intelligence (BI) (Clifton, 2012).

Embora muitas pessoas vejam o Google Analytics apenas como uma ferramenta de monitoramento de tráfego, ela pode funcionar como uma poderosa ferramenta no suporte a tomada de decisões (Plaza, 2011). Através da mineração e agrupamento dos dados e relatórios gerados pela ferramenta, é possível entender em quais atividades ou funcionalidade há maior carência de qualidade. Portanto, este serviço amplifica as possibilidades de gestão dos cursos de educação a distância.

Baseando-se nas funcionalidades disponíveis pelo Google Analytics, as informações definidas para nosso estudo foram as seguintes: dados sobre o AVA, dados sobre acesso de usuários por curso e informações gerais sobre os acessos. Além dessas informações, outras características como acessos por disciplina foram também definidas para análise em trabalhos futuros e não serão incluídas no escopo deste trabalho.

\subsection{Classificação dos dados}

Os dados coletados se referem ao período 2013.2 da UFPB Virtual. Esse período abrange desde o dia 19 de agosto até o dia 16 de dezembro de 2013.

Para a análise dos dados por curso deste estudo, foram escolhidas as licenciaturas a distância em Letras e Matemática, pois representam áreas de conhecimento distintas e são, juntamente com Pedagogia, os cursos pioneiros e com mais alunos da UFPB Virtual. Vale salientar que, neste trabalho, os dados de acesso por curso correspondem ao número de visitas feitas às páginas iniciais das disciplinas do curso no Moodle.

É importante dizer que a quantidade de visualizações é de todos os usuários e não apenas de alunos. Entretanto, como a quantidade de alunos é predominante maior as informações as inferências sobre um determinado conjunto de dados continuam válidas. ferramenta de BI.

$\mathrm{Na}$ próxima seção discutiremos as diferentes análises realizadas com a 


\section{Análise dos Dados}

Nesta seção, apresentamos uma análise dos dados coletados para esta pesquisa. Os dados foram agrupados em 3 tipos: acessos gerais, dados sobre a plataforma de acesso ao AVA e acessos por curso.

\subsection{Acessos Gerais}

A Figura 1 abaixo apresenta o número de acessos durante uma semana no meio do período 2013.2, de 07 a 13 de outubro. Percebe-se pelo gráfico que há um padrão de acessos durante a semana, onde diariamente há três picos evidentes de acesso: um primeiro pico pela manhã às $09 \mathrm{~h} 00$, um pico no turno da tarde às $14 \mathrm{~h} 00$, e um terceiro e maior pico a noite às $20 \mathrm{~h} 00$. Estes picos se repetem praticamente toda semana, com algumas pequenas variações no final de semana. Por exemplo, no domingo os picos são $10 \mathrm{~h} 00$ pela manhã, $17 \mathrm{~h} 00$ a tarde e $21 \mathrm{~h} 00$ a noite. Além disso, podemos também perceber no gráfico que os acessos vão diminuindo gradativamente da segunda-feira para o sábado, tendo uma elevação novamente ao domingo. Para reforçar esta evidência, o gráfico da Figura 2 apresenta o número de acessos por dias da semana ao longo de todo o período. Percebe-se que o dia realmente que tem o maior pico são as segundasfeiras e o dia com a menor quantidade de acessos é sempre o sábado.

Essa informação permite a alocação de tutores em horários estratégicos para melhor atender os alunos, por exemplo, através de plantões de atendimento via chat nos horários de pico. Apesar desses dados representarem todos os acessos ao AVA, eles podem ser restringidos para um curso específico, ajudando em estratégias mais direcionadas a realidade de cada um destes cursos.

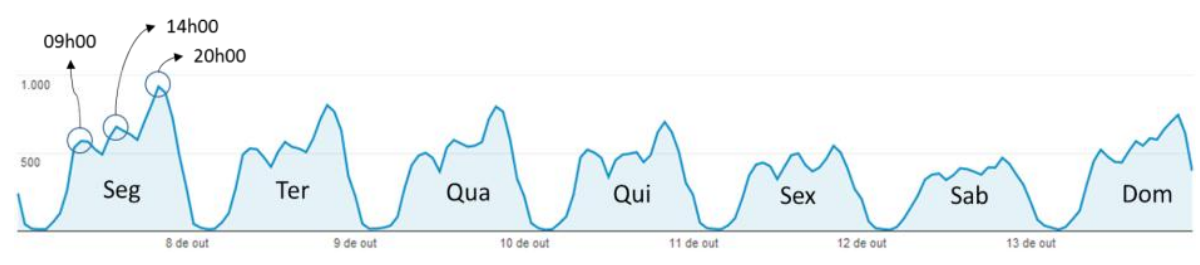

Figura 1 - Número de acessos por hora em uma semana

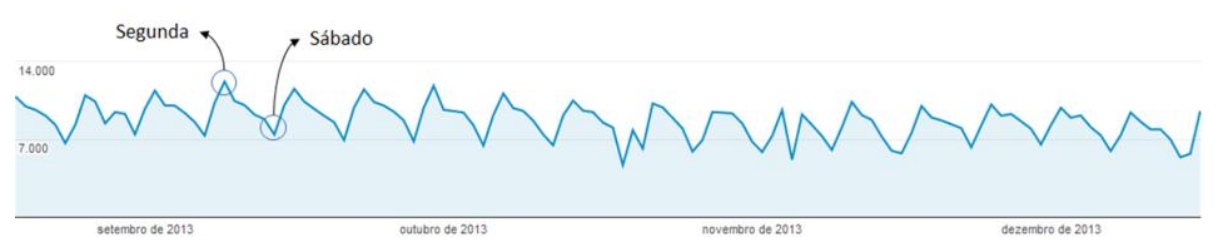

Figura 2 - Número de acessos por dia ao longo de todo o período letivo

O gráfico da Figura 3 mostra a quantidade de acessos ao AVA por semana em todo período letivo. Verifica-se uma leve descida no mês de novembro, ou seja, três quartos do período analisado. Depois disso, o número de acessos começa a subir novamente mas não chega ao seu valor de início do período. Este resultado pode evidenciar possíveis evasões ao longo do curso. É importante discutir ações para tentar minimizar o declínio da quantidade de acessos por parte dos usuários. 


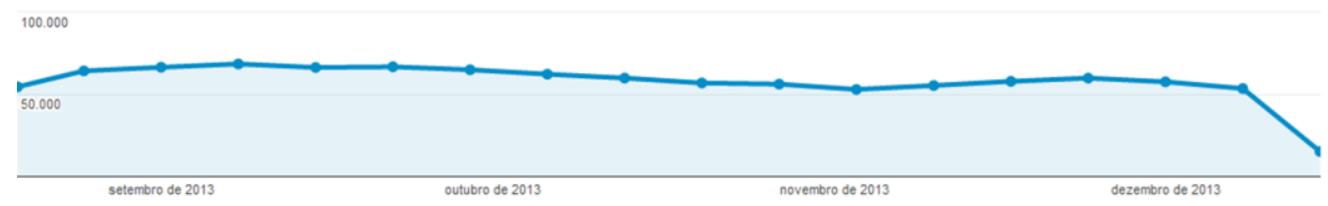

Figura 3 - Número de acessos por semana

\subsection{Dados sobre a plataforma}

Uma outra informação que é possível obter é o ranking dos navegadores utilizados para acessar o AVA. A Figura 4 apresenta estes dados do período em questão. Percebemos que a maioria dos usuários estão utilizando o Chrome como navegador. O segundo lugar ficou com o navegador Firefox. Percebe-se também o surgimento de navegadores específicos de smartphones, sugerindo que o acesso através destes dispositivos móveis já é uma realidade. Esta informação é importante uma vez que muitas vezes alguns recursos não funcionam de maneira correta em alguns navegadores. Portanto, é uma informação estratégia para a gestão do AVA considerar que navegadores estão sendo utilizados pelos usuários e dedicar esforços para resolver possíveis problemas de incompatibilidade destes.

\begin{tabular}{lr|l}
\multicolumn{1}{c}{ Navegador } & Sessōes & $\begin{array}{l}\text { Porcentagem de } \\
\text { Sessōes }\end{array}$ \\
\hline 1. Chrome & 577.160 & \multicolumn{1}{c}{$55,61 \%$} \\
\hline 2. Firefox & 337.654 & $32,53 \%$ \\
\hline 3. Internet Explorer & 90.614 & $8,73 \%$ \\
\hline 4. Android Browser & 11.616 & $1,12 \%$ \\
\hline 5. Safari & 9.652 & $0,93 \%$ \\
\hline 6. Mozilla & 3.746 & $0,36 \%$ \\
\hline 7. Opera & 3.067 & $0,30 \%$ \\
\hline 8. Opera Mini & 2.347 & $0,23 \%$ \\
\hline 9. S40 Ovi Browser & 514 & $0,05 \%$ \\
\hline 10. Nokia Browser & 301 & $0,03 \%$ \\
\hline
\end{tabular}

Figura 4 - Quantidade de acessos por navegador

A Figura 5 apresenta a quantidade de acessos com sua respectiva porcentagem por sistema operacional. Percebe-se que a grande maioria utiliza o sistema Windows. Os dados mostram a pouca utilização do Linux que é um software livre e tão incentivado pelo governo federal. Como os polos da UFPB Virtual são de responsabilidade da prefeitura do município, esta informação mostra que esses municípios precisam de um incentivo para adoção do sistema Linux.

\begin{tabular}{lr|l}
\multicolumn{1}{|c}{ Sistema operacional } & Sessöes & $\begin{array}{l}\text { Porcentagem de } \\
\text { Sessốes }\end{array}$ \\
\hline 1. Windows & 964.718 & \\
\hline 2. Linux & 37.835 & $\mid 3,95 \%$ \\
\hline 3. Android & 19.459 & $\mathbf{1}, 87 \%$ \\
\hline 4. iOS & 7.466 & $0,72 \%$ \\
\hline 5. Macintosh & 5.040 & $0,49 \%$ \\
\hline 6. (not set) & 1.431 & $0,14 \%$ \\
\hline 7. Windows Phone & 744 & $0,07 \%$ \\
\hline 8. Series40 & 514 & $0,05 \%$ \\
\hline 9. SymbianOS & 379 & $0,04 \%$ \\
\hline 10. Nokia & 149 & $0,01 \%$
\end{tabular}




\section{Figura 5 - Quantidade de acessos por sistema operacional}

O resultado apresentado na Figura 6 evidencia que o AVA já está sendo acessado via dispositivos móveis. Apesar de serem ainda a minoria, com apenas 3\% dos acessos, achamos que é uma tendência aumentar, principalmente quando o AVA permitir um melhor acesso para estes tipos de dispositivos.

A taxa de rejeição é a porcentagem de visitas a uma única página, ou seja, visitas nas quais o usuário sai de seu site na mesma página de entrada, sem interagir com essa. Percebemos que a taxa de rejeição de smartphones ainda é bastante alta, o que sugere que o nosso AVA ainda não tem uma estrutura adequada para ser utilizada por estes dispositivos. Por outro lado, a taxa de rejeição via tablets é baixa, uma vez que estes dispositivos possuem navegadores que conseguem acessar normalmente o AVA. Esta informação foi um fator motivador para o início de um projeto de desenvolvimento de uma aplicação mobile para rodar em Android.

A quantidade de páginas por sessão e a duração média da sessão indicam, respectivamente, o número de páginas que o usuário acessou dentro de uma mesma sessão e o tempo que durou esta sessão. Os dados confirmam o que foi explicado no parágrafo anterior onde percebe-se que usuários de smartphones não se mantém conectado ao AVA por muito tempo.

\begin{tabular}{|c|c|c|c|c|c|c|}
\hline \multirow[b]{2}{*}{ Categoria do dispositivo ? } & \multicolumn{3}{|l|}{ Aquisição } & \multicolumn{3}{|c|}{ Comportamento } \\
\hline & Sessões ? $\downarrow$ & $\begin{array}{c}\text { Porcentagem } \\
\text { de novas } \\
\text { sessôes } \\
?\end{array}$ & $\begin{array}{c}\text { Movos usuários } \\
?\end{array}$ & $\begin{array}{l}\text { Taxa de } \\
\text { rejeição } \\
?\end{array}$ & $\begin{array}{c}\text { Páginas I } \\
\text { sessão } \\
?\end{array}$ & $\begin{array}{c}\text { Duração } \\
\text { média da } \\
\text { sessão ? }\end{array}$ \\
\hline & $\begin{array}{r}1.037 .850 \\
\text { Porcentagem do total: } \\
100,00 \%(1.037 .850)\end{array}$ & $\begin{array}{r}9,39 \% \\
\text { Média do site: } \\
9,32 \%(0,69 \%)\end{array}$ & $\begin{array}{r}97.442 \\
\text { Porcentagem do } \\
\text { total: } 100,69 \% \\
(96.774)\end{array}$ & $\begin{array}{r}3,89 \% \\
\text { Média do } \\
\text { site: } 3,89 \% \\
(0,00 \%)\end{array}$ & $\begin{array}{r}20,32 \\
\text { Média do } \\
\text { site: } 20,32 \\
(0,00 \%)\end{array}$ & $\begin{array}{r}00: 21: 58 \\
\text { Média do site: } \\
00: 21: 58 \\
(0,00 \%)\end{array}$ \\
\hline 1. desktop & $1.007 .632(97,09 \%)$ & $9,25 \%$ & $93.165(95,61 \%)$ & $3,19 \%$ & 20,67 & $00: 22: 21$ \\
\hline 2. mobile & $18.674(1,80 \%)$ & $17,27 \%$ & $3.225(3,31 \%)$ & $40,51 \%$ & 5,88 & 00:07:25 \\
\hline 3. tablet & $11.544(1,11 \%)$ & $9,11 \%$ & $1.052(1,08 \%)$ & $5,51 \%$ & 12,70 & $00: 11: 33$ \\
\hline
\end{tabular}

Figura 6 - Quantidade de acessos por plataforma

A Figura 7 apresenta algumas informações de acesso apenas de usuários que acessaram de um dispositivo móvel.

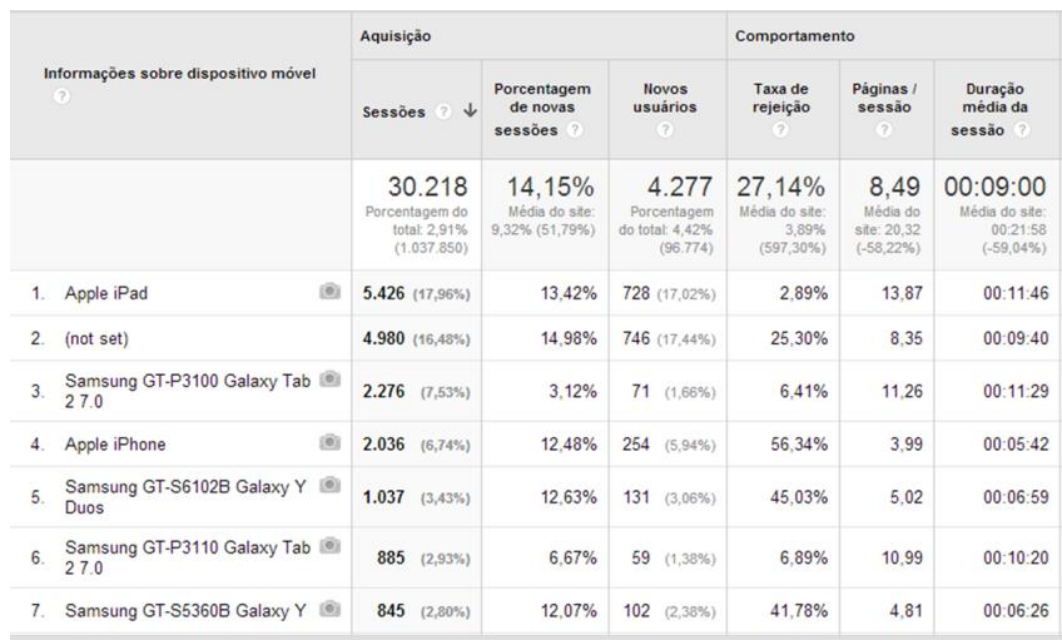


Figura 7 - Quantidade de acessos por dispositivo móvel

\subsection{Acessos por curso}

Os acessos por curso correspondem ao número de visualizações apenas das páginas que pertencem ao curso em análise.

A Figura 8 mostra o mesmo gráfico do número de visualizações da Figura 1, na semana de 07 a 10 de outubro de 2013, mas relativo aos cursos de Letras e Pedagogia, que possuem quantidades de alunos ativos muito próximas (1407 e 1438, respectivamente). Percebe-se a partir do gráfico que as linhas dos dois cursos são consideravelmente similares, com as curvas e variações praticamente sobrepostas umas com as outras. Essa semelhança indica que os acessos dos alunos de Letras e Pedagogia às páginas de seus cursos são muito parecidos ao longo das horas e dias, reforçando o padrão identificado.

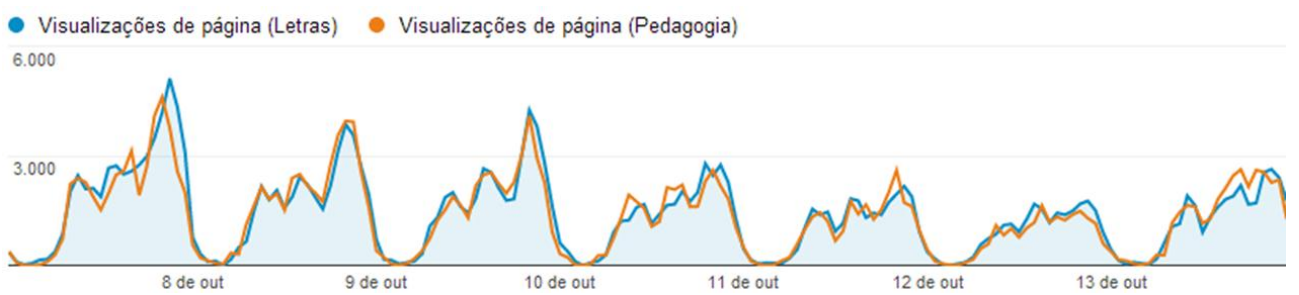

Figura 8 - Quantidade de acessos por hora em uma semana dos cursos de

\section{Letras e Pedagogia}

Além disso, é possível notar que os horários dos picos da Figura 8 também coincidem com os horários vistos na Figura 1, isto é, às 09h00, 14 h00 e 20h00 nos dias de semana, com pequenas variações. Ou seja, os horários de pico nos acessos às páginas dos cursos de Letras e Pedagogia coincidem com os horários de pico nos acessos a todo o AVA. A diminuição gradativa do acesso ao longo da semana até o sábado também ocorre nesses cursos.

Uma concentração maior de tutores nesses horários de pico e nos primeiros dias da semana parece, portanto, ser uma boa estratégia para os cursos de Letras e Pedagogia da UFPB Virtual.

A Figura 9 a seguir exibe o mesmo gráfico semanal anterior para os cursos de Matemática e Computação. O número menor de alunos ativos de Computação (467) em relação a Matemática (800) explica o fato de as curvas não estarem próximas. Mesmo assim, elas são bastante semelhantes, pois não existe um pico proeminente de visualizações à noite que se repete diariamente, como acontece nos cursos vistos anteriormente na Figura 8. Em vez disso, os acessos a esses cursos são mais distribuídos ao longo das horas úteis do dia, com picos em determinadas horas da manhã, tarde e noite.
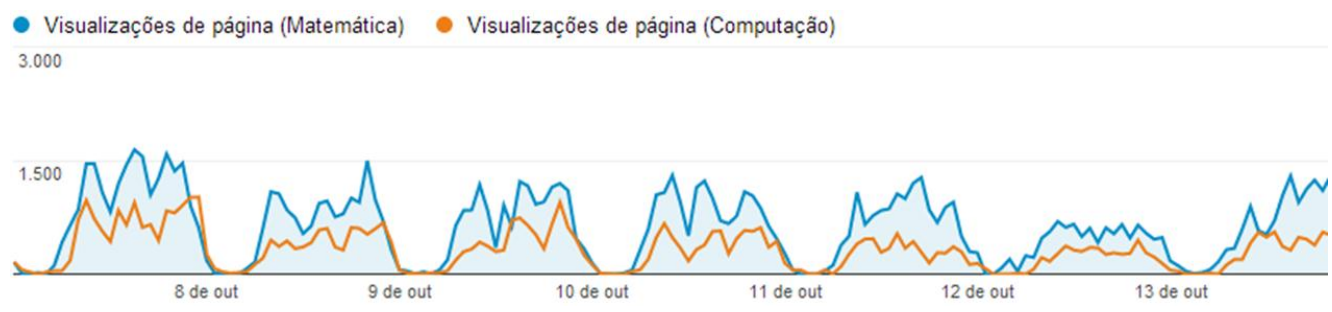
Figura 9 - Quantidade de acessos por hora em uma semana dos cursos de Licenciatura em Computação e Licenciatura em Matemática

Esse dado indica que, para os cursos de Matemática e Computação, uma maior distribuição dos horários de atendimentos dos tutores ao longo da semana pode ser mais inteligente e eficaz que concentrá-los em determinados horários.

É importante destacar o fato de os dois cursos da área de ciências humanas analisados (Letras e Pedagogia) terem padrões de acesso similares entre si, assim como são parecidos os padrões dos dois cursos da área de ciências exatas mostrados (Matemática e Computação). Essa informação pode ajudar a direcionar as estratégias de alocação de tutores nos plantões a partir das áreas de conhecimento, o que pode beneficiar futuros cursos que porventura sejam oferecidos na UFPB Virtual e que, por serem novos, carecem de dados de períodos anteriores cursados. Uma pesquisa mais aprofundada e qualitativa está sendo realizada para tentar levantar possíveis hipóteses que expliquem a fuga do padrão dos cursos de Matemática e Computação. Uma possível hipótese é a forma com que os professores organizam as salas virtuais no AVA.

Ao estender o intervalo dos dados de visualizações de páginas para todo o período letivo de 2013.2 e agrupar esses acessos por semana, novas análises podem ser feitas. A Figura 10 apresenta o gráfico semanal dos cursos de Letras e Pedagogia ao longo de 2013.2. Assim como visto na Figura 3, nota-se um maior número de acessos nas primeiras semanas do período. A quarta semana (08 a 14 de setembro) foi o intervalo que apresentou a maior quantidade de acessos de todo o período letivo para ambos os cursos. A partir da quinta semana, que vai de 15 a 21 de setembro, a tendência geral dos acessos é de queda até o começo de novembro. Após isso, os acessos semanais passam a crescer até as últimas semanas antes do término do período letivo.

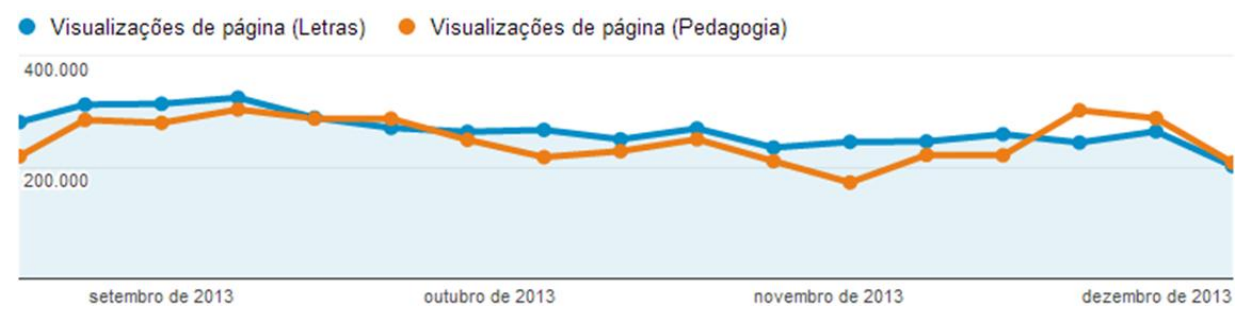

Figura 10 - Acessos por semana às páginas de Letras e Pedagogia em 2013.2

No caso do curso de Pedagogia, que possui curvas mais acentuadas que as de Letras, a semana com mais acessos (08 a 14 de setembro) registrou 303 mil visualizações, enquanto que a semana com menos acessos (03 a 11 de novembro) contabilizou 172 mil visualizações, uma diminuição de mais de 43\%.

A Figura 11 mostra o gráfico do período agrupado por semana para os cursos de Matemática e Computação. Assim como na Figura 10, os maiores números de acessos são vistos nas primeiras semanas, após as quais a tendência é de queda até novembro.

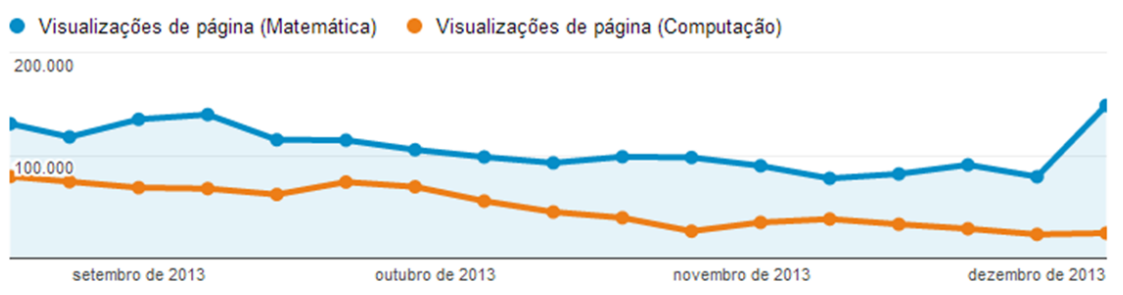




\section{Figura 11 - Acessos por semana às páginas de Matemática e Computação em 2013.2}

A linha do curso de Computação mostra uma diminuição mais constante ao longo de toda a extensão do período. Para este curso, a semana em que houve mais acessos (primeira semana) contou 79 mil visualizações, enquanto que a semana com menos acessos (penúltima semana) teve 23 mil visualizações, uma diminuição preocupante de mais de $70 \%$.

Apesar das linhas dos quatro cursos analisados terem suas particularidades, a queda constante do acesso, que se inicia a partir de meados de setembro e se estende por quase dois meses, até o começo de novembro, parece indicar um gradativo desleixo ou falta de atenção dos alunos em torno do curso ao longo do período. Quando o final do período se aproxima, os estudantes voltam a acessar a plataforma com mais frequência, provavelmente para recuperar o ritmo do curso e evitar reprovações em disciplinas.

A partir dessa hipótese, é possível apontar estratégias de tutoria para os cursos no âmbito do período letivo. Um reforço e atenção maiores dos tutores e professores durante o intervalo de queda dos acessos podem ajudar os alunos a se manterem constantemente ativos na plataforma e, portanto, alinhados com o andamento das disciplinas, o que evitaria essa "corrida" de fim do período em busca de recuperar as semanas ausentes.

\section{Trabalhos relacionados}

O trabalho de Weirich, Gasparini \& Kemczinski (2007) mostra uma ferramenta de análise de log do Moodle (AdaptWeb) que foi implementada para obter informações referente ao comportamento do aluno frente ao ambiente virtual de aprendizagem. Plaza (2011) apresenta a análise dos acessos em um site de turismo a partir do Google Analytics visando ações de marketing e publicidade a partir dos perfis do usuário. Assim, o presente trabalho tem como aspecto inovador o uso do Google Analytics aplicado à Educação.

\section{Considerações Finais e Trabalhos Futuros}

Uma das principais contribuições deste trabalho é promover um entendimento mais analítico sobre os padrões de acesso aos ambientes virtuais de aprendizagem. Esta análise visa diminuir das barreiras existentes no que se refere ao ensino a distância. Portanto, este trabalho torna-se relevante uma vez que apresenta como a realização de análises, através de uma ferramenta de Business Intelligence, pode melhorar a utilização dos recursos nos cursos a distância, melhorando a eficiência na gestão destes.

O Google Analytics foi utilizado com ferramenta de BI e permite gerar diversas informações relacionadas aos acessos dos usuários ao AVA, tais como: quantidade de acessos por hora, dia, semana, mês ou ano; quantidade de acessos por sistema operacional e por plataforma; quantidade de acessos por navegador; taxas de rejeição e de saída de uma página; duração média da sessão; entre outras.

Apesar dos bons resultados encontrados nessa etapa inicial, são necessárias pesquisas complementares para entender as causas referentes aos resultados obtidos. Através destas pesquisas será possível propor melhorias significantes nos cursos. Dentre os trabalhos futuros, serão realizadas pesquisas com apoio de metodologias qualitativas, 
para aprofundar e entender melhor os resultados trazidos nas análises iniciais, como por exemplo, relacionar estes resultados com o perfil do corpo discente e com as metodologias adotadas pelos professores dos diferentes cursos.

\section{Referências}

LITTO, F. M.; FORMIGA, M. M. Educação à distância: o estado da arte. São Paulo, Pearson, 2009.

ABED. Associação Brasileira de Educação A Distância. Censo EAD.BR. Relatório Analítico da aprendizagem a distância no Brasil. 2012. Disponível em: http://www.abed.org.br/censoead/censo2012.pdf. Acesso em 30 de abril de 2014.

SANTOS, N. Estado da arte em espaços virtuais de ensino e aprendizagem. Revista Brasileira de Informática na Educação, Florianópolis, SBC, n.4, abril, 1999.

BARBERA, ELENA. Quality in Virtual Education Environments. British Journal of Educational Technology, v35.1, p. 13-20, 2004

DILLENBOURG, P.; SCHNEIDER, D.; SYNTETA, P.. Virtual Learning Environments. 3rd Hellenic Conference Information \& Communication Technologies in Education, 2002

WICKERSHAM, L. E., DOOLEY, K.E.. A content analysis of critical thinking skills as an indicator of quality of online discussion in virtual learning communities, The Quarterly Review of Distance Education, v7.2, p.185-193. 2006.

CHAMLIAN, H. C.. Docência na universidade: professores inovadores na USP. Cadernos de pesquisa, 118, São Paulo, 2003.

IKE-ELECHI O. S.; NEIL AND F., C. N. Predicting students attitudes towards advertising on a university Virtual Learning Environment (VLE). Active Learning in Higher Education. V. 13.1. p. 63-75, 2012.

BEETHAM, H., SHARPE, R. Rethinking pedagogy for a digital age: Designing and delivering e-learning, $2^{\mathrm{a}}$ ediç̧ão, 2007

CLIFTON, B. Advanced Web Metrics with Google Analytics, 3rd Edition, Abril, 2012

PLAZA B., Google analytics for measuring website performance. Tourism Management, V. 32.3, p. 477-481, 2011. 